\title{
IMMIGRATION ET TERRORISME : « CORRÉLATION MAGIQUE »ET INSTRUMENTALISATION POLITIQUE
}

\author{
Vincent Geisser
}

Centre d'Information et d'Etudes sur les Migrations Internationales | "Migrations Société »

2020/4 N 182 | pages 3 à 13

ISSN 0995-7367

Article disponible en ligne à l'adresse :

https://www.cairn.info/revue-migrations-societe-2020-4-page-3.htm

Distribution électronique Cairn.info pour Centre d'Information et d'Etudes sur les Migrations Internationales.

(C) Centre d'Information et d'Etudes sur les Migrations Internationales. Tous droits réservés pour tous pays.

La reproduction ou représentation de cet article, notamment par photocopie, n'est autorisée que dans les limites des conditions générales d'utilisation du site ou, le cas échéant, des conditions générales de la licence souscrite par votre établissement. Toute autre reproduction ou représentation, en tout ou partie, sous quelque forme et de quelque manière que ce soit, est interdite sauf accord préalable et écrit de l'éditeur, en dehors des cas prévus par la législation en vigueur en France. Il est précisé que son stockage dans une base de données est également interdit. 


\title{
Immigration et terrorisme : « corrélation magique » et instrumentalisation politique
}

\author{
Vincent GEISSER
}

« Terrorisme et immigration. Depuis plusieurs jours, les deux mots se mêlent dans les discours politiques $\aleph^{\mathrm{I}}$. Ce constat dressé par Julia Pascual, journaliste au quotidien Le Monde, juste après les attentats dramatiques de Nice (29 octobre) $)^{2}$ et de Vienne (2 novembre) $)^{3}$, n'est ni nouveau ni propre au cas français. En effet, l'association du fait migratoire au phénomène terroriste constitue une figure classique de la rhétorique des partis d'extrême droite et des mouvements populistes. En novembre 2015, dans un entretien au média américain Politico, le Premier ministre hongrois, Viktor Orbán, déclare ainsi : «bien sûr, ce n'est généralement pas admis, mais le fait est que tous les terroristes sont essentiellement des migrants. La question est de savoir quand ils ont migré vers l'Union européenne $»^{4}$. Ce type de déclaration n'est pas purement conjoncturel : il s'inscrit dans une longue dérive sécuritaire et identitaire du gouvernement hongrois qui joue sur les registres anti-immigrés et xénophobe pour faire oublier aux électeurs les dégâts moraux et sociaux suscités par une politique de restructuration néolibérale particulièrement brutale pour la société : « Si, récemment, l'attitude autoritaire et sécuritaire du gouvernement a été justifiée par la présence des réfugiés, les processus de militarisation et de sécurisation de la société s'inscrivent en réalité dans une temporalité plus longue. Depuis son arrivée au pouvoir en 20Io, le Premier ministre Viktor Orbán a fait adopter une série de mesures répressives sous prétexte de protéger l'ordre social et moral contre des figures d'altérité, d'abord intérieures - en premier lieu les Roms et les sans-abri »5. Dans la Hongrie post-communiste, l'association rhétorique entre terrorisme et immigration participe donc d'une politique de

1. PASCUAL, Julia, "Le lien entre terrorisme et immigration à l'épreuve des faits", Le Monde, 13 novembre 2020.

2. FANSTEN, Emmanuel; FRÉNOIS, Mathilde; LEBOUCQ, Fabien, "Nice : trois morts dans I'attaque terroriste contre la basilique Notre-Dame", Libération, 29 octobre 2020.

3. "Autriche : au moins quatre morts dans une 'attaque terroriste' à Vienne", Le Monde, 2 novembre 2020.

4. KAMINSKI, Matthew, "Viktor Orbán: 'All the terrorists are migrants'" [En ligne], Politico, November 23, 2015, https://www.politico.eu/article/viktor-orban-interview-terroristsmigrants-eu-russia-putin-borders-schengen. [Traduction de l'auteur].

5. CANTAT, Céline, "La Hongrie, chien de garde de l'Europe", Plein droit, $\mathrm{n}^{\circ} 111$, décembre 2016, pp. 19-22. 
criminalisation de toutes les figures de l'altérité jugées « déviantes » par rapport à un idéal de pureté magyare ${ }^{6}$.

En France, depuis le début des années 2000, il est fréquent que les responsables locaux et nationaux du Front national - devenu en 2018 le Rassemblement national (RN) - établissent une causalité entre la présence des migrants et des descendants d'immigrés sur le territoire français et le terrorisme. Ainsi, en 20I2, en pleine campagne pour l'élection présidentielle, Marine Le Pen faisait un lien direct entre les actes terroristes commis en région toulousaine par Mohamed Merah et le «problème de l'immigration » en général: "Combien de Mohamed Merah dans les bateaux, les avions, qui chaque jour arrivent en France remplis d'immigrés?» "Combien de Mohamed Merah parmi les enfants de ces immigrés non assimilés ? [...] Mohamed Merah n'est peut-être que la partie émergée de l'iceberg " ${ }^{7}$. Nos propres observations sociologiques conduites à Marseille durant la campagne électorale des municipales en mars-juin 2020 montrent que le responsable local du RN, Stéphane Ravier, a recours de manière récurrente à cet amalgame anxiogène « immigrés = terroristes " pour mobiliser certaines franges effrayées de son électorat ${ }^{8}$. Plus récemment, le député européen RN, Jérôme Rivière, a adressé une question écrite au président du Conseil de l'Europe, lui demandant de reconnaître explicitement le lien direct entre « immigration » et « terrorisme » : "Le Conseil considère$t$-il toujours qu'il n'y a aucun lien entre terrorisme et immigration? Le Conseil est-il prêt à suspendre l'espace Schengen et à laisser aux États la liberté de contrôler leurs frontières pour faire face à une menace mortelle pour les pays de l'Europe?»?.

Au-delà de l'actualité dramatique, cette association discursive entre terrorisme et immigration a des racines historiques profondes : elle constitue l'un des fondamentaux de l'imaginaire politique xénophobe de l'extrême droite. De l'Action française de Charles Maurras au régime de Vichy (I940-I944), en passant par les groupuscules Occident et Ordre nouveau, les actions terroristes ont toujours été imputées aux métèques, aux immigrés, aux minoritaires et aux Français d'origine étrangère, comme nous le rappelle le souvenir de l'Affiche rouge, sur laquelle les montages photographiques et les légendes

6. TAMÁS, Pál, "Hongrie : à défaut d'immigrés, on vise les Roms", Alternatives internationales, $n^{\circ} 52$, septembre 2011, p. 40.

7. AFP, "Marine Le Pen fait I'amalgame entre immigration et terrorisme" [En ligne], Libération, 25 mars 2012, https://www.liberation.fr/france/2012/03/25/marine-le-pen-fait-I-amalgameentre-immigration-et-terrorisme_805592.

8. GEISSER, Vincent, Carnets de terrain, Marseille, mars-juin 2020. Voir l'intégralité du discours de Stéphane Ravier, tête de liste RN aux élections municipales de Marseille, meeting au Parc Chanot, 6 mars 2020, https://www.youtube.com/watch?v=TRkQ9cuz3z4.

9. RIVIÈRE, Jérôme, député européen, membre du Rassemblement national, communiqué du 10 novembre 2020, https://rassemblementnational.fr/communiques/terrorisme-et-immigration-leconseil-de-leurope-doit-abandonner-schengen. 
mettaient en exergue les origines juive, arménienne, espagnole, hongroise, italienne, polonaise, etc. desdits « terroristes $»^{\mathrm{IO}}$.

Bien sûr, cette " corrélation magique » entre "immigration » et « terrorisme » n'est pas exclusive de l'extrême droite et encore moins du contexte européen. Les travaux d'Ayse Ceyhan, maître de conférences à Sciences Po et spécialiste des technologies de surveillance et de sécurité, montrent comment les attentats du ir septembre 200I ont conforté chez les responsables politiques et les décideurs publics étatsuniens l'idée d'une corrélation entre terrorisme et immigration, jouant doublement sur la figure de l'ennemi intérieur et de l'ennemi extérieur, les deux étant supposées être reliées par une commune ascendance immigrée: "Les attentats tragiques du II septembre $200 \mathrm{I}$ ont eu pour conséquence d'établir une association entre terrorisme et immigration, de mettre en avant la sécurité de la patrie et de soulever la question de l'allégeance des citoyens d'origine étrangère envers les États-Unis. [...] Des rhétoriques sur l'ennemi intérieur reprenant les métaphores de la cinquième colonne ou de la tumeur cancérogène reviennent dans les discours qui font pression sur les communautés pour qu'elles expriment leur loyauté envers les États-Unis ${ }^{\mathrm{II}}$. Très souvent d'ailleurs, comme le note Ayse Ceyhan, les discours publics finissent par opérer un glissement de l'immigré clandestin vers l'immigré légal, les deux étant présentés comme les deux faces d'une même "pièce terroriste » : «La loi Patriot établit clairement un lien entre terrorisme et immigration. Mais il convient de remarquer que cette association ne vise plus les clandestins comme c'était le cas jusque-là dans les discours et législations sécuritaires. Cette fois-ci les cibles sont les étrangers entrés sur le territoire des États-Unis avec un visa en règle fourni par un des consulats américains au Moyen-Orient ou en Europe $»^{\mathrm{I}}$. De tels discours anxiogènes contribuent en dernier ressort à conforter une théorie de sens commun accréditant l'existence d'un "continuum de menaces» pour la sécurité nationale : l'immigré illégal, le migrant établi, le descendant d'immigré, le national d'origine étrangère et, au bout de la chaîne, les acteurs de la solidarité avec les immigrés et les minorités, suspectés de déloyauté patriotique : «Dans ce contexte de suspicion, les discours sur l'ennemi évoquent plus que jamais les allégeances micro communautaires et soulignent les menaces qu'elles peuvent faire peser sur l'État en terme de loyauté $»^{13}$.

\footnotetext{
10. COURTOIS, Stéphane; PESCHANSKI, Denis; RAYSKI, Adam, Le Sang de l'étranger: les immigrés de la MOI dans la Résistance, Paris : Éditions Fayard, 1989, 470 p. ; AMAT, Jorge ; PESCHANSKI, Denis, "À propos du documentaire. La Traque de l'affiche rouge", Matériaux pour l'histoire de notre temps, $\mathrm{n}^{\circ} 1-2,2008, \mathrm{pp}$. 55-63.

11. CEYHAN, Ayse, "Terrorisme, immigration et patriotisme. Les identités sous surveillance", Cultures \& conflits, $n^{\circ}$ 44, Hiver 2001, pp. 117-133.

12. CEYHAN, Ayse, ibid.

13. CEYHAN, Ayse, ibid.
} 
Toutefois, cette corrélation imaginaire entre immigration et terrorisme ne se limite pas aux contextes paroxystiques (les lendemains d'attentats et de violences collectives) : elle relève aussi d'une temporalité plus longue, celle d'un discours d'État, révélateur de l'enracinement d'une "gouvernementalité par l'inquiétude », désormais bien ancrée dans nos démocraties libérales - de plus en plus « illibérales » d'ailleurs - que l'on pourrait qualifier de « démocraties autoritaires ${ }^{I 4}$. Sur ce plan, les travaux du sociologue Didier Bigo analysent de manière très fine comment le thème de l'immigration devient un élément central du discours de sécurisation de l'espace public et, davantage encore, une pièce majeure des dispositifs de contrôle et de surveillance de nos sociétés démocratiques : "Le discours sécurisant l'immigration est alors en position de force symbolique et devient une technologie politique, une modalité de la gouvernementalité contemporaine. Bien que les ambiguïtés du discours de sécurité aient été souvent largement démontrées, le discours de sécurisation continue ${ }^{15}$. À l'échelon européen (UE) comme au niveau national, les registres sécuritaires de la « lutte contre le terrorisme » et de la « lutte contre l'immigration clandestine » se trouvent totalement imbriqués tant dans les représentations et les discours que dans les politiques publiques, où « la facilitation de l'immigration irrégulière est de plus associée à divers autres crimes et délits (terrorisme, trafic de drogues, d'armes) dans un phénomène de "polycriminalité », ce qui accentue également la perception de risques liés aux migrants $»^{16}$. La sécurisation de nos sociétés affecte directement les processus cognitifs et les modes de perception du phénomène migratoire, au point que l'immigration illégale dite « clandestine » devient une catégorie à part entière du traitement de la «menace terroriste ». Cette combinatoire sécuritaire est tellement prégnante dans les esprits des responsables politiques et des experts européens qu'ils ont de plus en plus tendance à l'énoncer spontanément, en recourant à un langage à la fois technocratique et statistique visant à légitimer auprès des opinions publiques la corrélation magique entre immigration et terrorisme. Le discours d'exception devient ainsi la norme d'une gouvernementalité par la peur qui conditionne tous les aspects de la vie quotidienne des migrants illégaux, des immigrés installés, des descendants de l'immigration et même des citoyens nationaux, selon le processus de glissement idéologique et symbolique décrit précédemment: "Seulement, quand un discours politique ne voit plus que l'inimitié, utilise les outils statistiques les plus divers à des fins de sécurité pour matérialiser l'adversaire, et invoque, avec plus ou moins

14. DABÈNE, Olivier; GEISSER, Vincent; MASSARDIER, Gilles, Autoritarismes démocratiques. Démocraties autoritaires au XXI siècle. Convergences Nord/Sud, Paris : Éditions La Découverte, 2008, $336 \mathrm{p}$.

15. BIGO, Didier, "Sécurité et immigration : vers une gouvernementalité par l'inquiétude ?", Cultures \& conflits, $n^{\circ} 31-32$, Automne-Hiver 1998, pp. 13-38.

16. PIQUET, Agathe, "Europol et la 'sécuritisation' des migrations irrégulières", Migrations société, $n^{\circ} 165$, juillet-septembre 2016, pp. 133-149. 
de pertinence, les relations entre ces grandes structures, leurs longs processus et les raisons de la nécessaire "sécurisation" de l'immigration, c'est qu'il est en train de définir une politique de maîtrise (des flux), d'insertion contrôlée, de surveillance (des mentalités et des attitudes). C'est qu'il est mû par une "rhétorique de la mise en péril" et qu'il devient un discours de "sécurisation" plaidant pour des solutions d'exception ${ }^{17}$.

Au-delà de la parole, il y a aussi les actes et les décisions. L'actualité de ces dernières semaines, marquée à la fois par une aggravation de la crise sanitaire due à l'épidémie de la Covid-I9, et à une montée du climat anxiogène consécutif aux attentats terroristes de Nice et de Vienne, éclaire ce processus de sécurisation par la mise en corrélation de la «question migratoire » avec la «question terroriste». Ce n'est pas un hasard si le ministre de l'Intérieur, Gérald Darmanin, a effectué au début du mois d'octobre 2020 une tournée en Italie, à Malte et dans les pays du Maghreb (Algérie, Maroc et Tunisie) pour expliquer la «nouvelle doctrine française $»^{18}$ en matière sécuritaire visant à traiter simultanément la question migratoire et la question terroriste par un contrôle renforcé des flux migratoires et un rapatriement des «radicalisés » dans leur pays d'origine ${ }^{\mathrm{I}}$. Dans une perspective similaire, le président de la République, Emmanuel Macron, a multiplié les gestes afin de « rassurer » les Français sur le fait que l'Hexagone n'avait pas vocation à devenir une terre d'accueil pour les terroristes étrangers. Outre un déplacement le 5 novembre 2020 à la frontière franco-espagnole suite à l'attentat de l'église Notre-Dame de Nice (le 29 octobre $)^{20}$, lors duquel il s'est largement exprimé sur la nécessité de renforcer les contrôles aux frontières et de réformer en profondeur l'espace Schengen, il a convoqué le io novembre un mini-sommet européen virtuel en présence du chancelier autrichien, Sebastian Kurz, de la chancelière allemande, Angela Merkel, du Premier ministre néerlandais, Mark Rutte, et de la présidente de la Commission européenne, Ursula Von der Leyen. Au terme du sommet, le président Macron a prononcé un discours qui, en dépit de quelques nuances, légitime une lecture sécuritaire de la question migratoire, recourant implicitement à la corrélation magique entre immigration et terrorisme. Sous couvert d'une certaine prudence rhétorique, pour ne pas

17. BIGO, Didier, "Sécurité et immigration : vers une gouvernementalité par l'inquiétude?", Cultures \& conflits, $\mathrm{n}^{\circ} 31-32$, Automne-Hiver 1998, pp. 13-38.

18. Nous mettons des guillemets car cette doctrine sécuritaire n'a en fait rien de nouveau. Elle $s^{\prime}$ inscrit dans la droite ligne des politiques migratoires pratiquées par les gouvernements français depuis trente ans.

19. AFP, "Darmanin salue à Alger la coopération sur la sécurité et la migration" [En ligne], 8 novembre 2020, https://www.lorientlejour.com/article/1240057/darmanin-salue-a-algerla-cooperation-sur-la-securite-et-la-migration.html.

20. "Macron à la frontière espagnole pour durcir le contrôle aux frontières" [En ligne], Le Figaro/AFP, 5 novembre 2020, https://www.lefigaro.fr/flash-actu/macron-a-la-frontiereespagnole-pour-durcir-les-controles-au-frontieres-20201105. 
donner des arguments à l'extrême droite ${ }^{21}$, le discours présidentiel conforte très largement l'idée reçue selon laquelle le développement du terrorisme en Europe serait dû, en grande partie, à une gestion trop laxiste de la liberté de circulation, appelant à une refonte totale de l'espace Schengen: "C'est essentiel, il ne faut en rien confondre la lutte contre l'immigration clandestine et le terrorisme, mais il nous faut regarder lucidement les liens qui existent entre ces deux phénomènes. L'attentat de Nice, malheureusement, en est aussi l'illustration. L'espace de libre circulation Schengen est un des principaux acquis de la construction européenne, mais il reposait, en contrepartie de la promesse de libre circulation sans frontières intérieures, sur une promesse de protection et de sécurisation de nos frontières extérieures. Cette seconde promesse n'a pas été suffisamment tenue, et les opinions publiques des États confrontés à la menace terroriste ne pourront longtemps accepter le maintien de nos frontières ouvertes si nous ne réformons pas en profondeur l'espace Schengen. Nous l'avons vu au printemps, dans le contexte pandémique, nous le voyons aujourd'hui en ce qui concerne le terrorisme $\aleph^{22}$. Sur ce plan, le président français s'en est pris directement à ce qu'il qualifie luimême de "dévoiement du droit d'asile », confortant ainsi la thèse de sens commun des «faux réfugiés » qui se glisseraient parmi les exilés pour mieux se livrer à toutes sortes de trafics ou de violences sur le territoire européen : «Dans tous nos pays, nous assistons à un dévoiement du droit d'asile [qui est utilisé] par des trafiquants, des réseaux ou des personnes venant de pays qui ne sont pas en guerre ${ }^{23}$. Au final, Emmanuel Macron en vient à proposer une solution sécuritaire qui accrédite non seulement la corrélation magique entre immigration et terrorisme mais, en plus, entretient l'illusion de la supériorité des technologies de surveillance comme remède miracle à la fois à l'immigration illégale et au terrorisme, à travers la création d'une nouvelle institution européenne : "Je suis favorable à cet égard que nous puissions mettre en place un véritable Conseil de sécurité intérieure. Nous aurons l'occasion, dans les prochains jours, de faire la proposition aux autres États ici présents, un projet de réforme et de le partager avec l'ensemble de nos collègues pour pouvoir avancer en la matière. Réformer Schengen, c'est permettre la liberté en sécurité $»^{24}$.

21. BOST, Olivier, "Immigration, islamisme, terrorisme: les nouvelles convictions d'Emmanuel Macron" [En ligne], RTL, 6 novembre 2020, https://www.rtl.fr/actu/politique/immigrationislamisme-terrorisme-les-nouvelles-convictions-d-emmanuel-macron-7800917960.

22. MACRON, Emmanuel, déclaration à la Conférence de presse du 10 novembre 2020 sur la réponse européenne à la menace terroriste, https://www.elysee.fr/front/pdf/elyseemodule-16543-fr.pdf.

23. BERDAH, Arthur, "Islamisme: Macron appelle à 'regarder lucidement les liens qui existent' entre immigration et terrorisme" [En ligne], Le Figaro, 10 novembre 2020, https://www.lefigaro.fr/politique/islamisme-macron-appelle-a-regarder-lucidement-lesliens-qui-existent-entre-immigration-et-terrorisme-20201110.

24. MACRON, Emmanuel, déclaration à la Conférence de presse du 10 novembre 2020 sur la réponse européenne à la menace terroriste, https://www.elysee.fr/front/pdf/elyseemodule-16543-fr.pdf. 
L'opposition de droite et d'extrême droite a surenchéri sur la relation entre immigration et terrorisme, ne s'embarrassant même pas de précautions oratoires, et présentant la corrélation magique comme une évidence sociologique. Le président des Républicains, Christian Jacob, n’a pas hésité à faire un lien direct entre présence étrangère en France et risque terroriste: «Depuis trois semaines: trois attentats, une tentative stoppée de justesse à Lyon. Quatre fois des étrangers et parfois accueillis soit par des titres de séjour soit comme mineur non accompagné. [...] Donc le problème de limmigration et son contrôle doit être posé. [...] il faudra faire évoluer les textes. Nous devons renforcer la législation et le contrôle sur l'immigration, ce que nous n'avons cessé de demander depuis 8 ans $»^{25}$. La présidente de la Région Île-de-France, Valérie Pécresse, s'est attaquée au droit d'asile, laissant sous-entendre que sa gestion laxiste par les différents gouvernements favoriserait le terrorisme sur le territoire national : «On ne peut plus aujourd'hui donner l'asile à nos ennemis $»^{26}$. Par ailleurs, elle s'en est prise aux immigrés résidents et aux descendants d'immigrés qui n'aimeraient pas la France, jouant ainsi à fond sur le fantasme de la déloyauté ou de la double allégeance: "Il y a des migrants pour qui la France c'est le pays rêvé, c'est le pays des Lumières, et qui aiment profondément la France (et) il y a ceux qui peuvent être nés en France, des Français, qui n'aiment pas leur propre pays et qui vont porter les armes contre lui. Nous devons aujourd'hui être beaucoup plus fermes ${ }^{27}$. Quant au Rassemblement national, par la voix du député européen Thierry Mariani, il s'est livré à une tentative de justification statistique de la relation entre immigration et terrorisme : "Le lien entre terrorisme et immigration est évident. Sur les 8000 personnes signalées comme radicalisées, plus de la moitié sont étrangers, 8II sont même clandestins, qu'attend-on pour les mettre dehors? Les Français veulent des actes, plus des mots ! ${ }^{28}$.

Que faire? Quelle pourrait être la réponse des chercheurs en sciences sociales à cette corrélation magique énoncée au fil des discours par les responsables politiques français et européens? Faut-il esquiver la question, par peur de légitimer les lectures populistes des phénomènes migratoires ? Convient-il, au contraire, de s'en saisir pour essayer de déconstruire les fantasmes qui surgissent autour du lien entre immigration et terrorisme?

25. Interview de Christian Jacob, "Le problème de l'immigration et son contrôle doit être posé", émission «6 minutes pour trancher» [En ligne], RTL, 30 octobre 2020, https://www.rtl.fr/actu/ politique/terrorisme-le-probleme-de-l-immigration-et-son-controledoit-etre-pose-dit-jacob-7800914135.

26. LEPELLETIER, Pierre, "Terrorisme islamiste : Pécresse appelle 'les musulmans' à déclarer 'd'une voix forte que ce n'est pas en leur nom'" [En ligne], Le Figaro, 30 octobre 2020, https://www.lefigaro.fr/politique/terrorisme-islamiste-pecresse-appelle-les-musulmansa-declarer-d-une-voix-forte-que-ce-n-est-pas-en-leur-nom-20201030.

27. Ibid.

28. Propos tenus par Thierry Mariani le 17 octobre 2020 sur la chaîne CNews, cités par PASCUAL, Julia, "Le lien entre terrorisme et immigration à l'épreuve des faits", Le Monde, 13 novembre 2020 , art. cité. 
Dans un article fort stimulant publié par la revue Hommes \& migrations dans le contexte des émotions suscitées par l'attentat du Bataclan en novembre 20I5, Jean-Baptiste Meyer, sociologue et directeur de recherches à l'Institut de recherche pour le développement (IRD) appelle à « détabouiser » le débat, afin de ne pas laisser le monopole à la "pensée magique» et de réfléchir avec les outils des sciences sociales à l'hypothèse d'une relation entre immigration et terrorisme. Il écrit ainsi : "Car il y a bien un lien entre la migration et ce terrorisme; un lien empirique et visible... Le nier aujourd'hui reviendrait à renoncer à expliquer une part importante de ces événements. Et, ce faisant, à donner un surcroît de crédibilité à ceux qui, dans des amalgames tronqués mais concrets, forgent les fers de la discorde $»^{29}$. Même si nous ne partageons pas forcément toutes les conclusions de l'auteur, reconnaissons avec lui que la «dimension migratoire» est bien présente dans le phénomène terroriste actuel, ne serait-ce que dans les parcours biographiques de certains activistes jihadistes, et surtout dans leur imaginaire, car la grande majorité d'entre eux rêvent d'accomplir la hijra (terme signifiant en arabe « immigration » ou « exil» en référence à la mobilité du Prophète Mohamed en 622 de la Mecque à Médine), afin de contribuer à la construction et au triomphe de l'État islamique. À cet égard, nous ne pouvons qu'être d'accord avec Jean-Baptiste Meyer : l'imaginaire jihadiste renvoie à un projet à la fois religieux et idéologique mais aussi migratoire qui induit nécessairement des mobilités humaines. Comme l'écrit le politiste Mohamed-Ali Adraoui qui, dans le cadre de sa recherche doctorale, a enquêté plusieurs années sur des jeunes salafistes français, "la hijra témoigne d'une "esthétique" de l'existence, d'un certain rapport à la société française, d'une valorisation de l'Orient et d'une forme d'engagement politique (bien que le quiétisme salafiste abhorre le militantisme organisé), ainsi que d'une mondialisation des marges s'opérant aussi bien à l'ombre des États qu'en opposition à eux; au premier rang desquels la France qui paradoxalement leur fournit la nationalité leur permettant de voyager en "terre dislam" dans le but de s'extirper du modèle qu'elle représente. En mettant la focale sur la hijra, puis en la resituant dans le déroulement des carrières salafistes, il devient ainsi possible de figurer certains des ressorts globaux de l'identification à un certain fondamentalisme contemporain $»^{30}$.

En revanche, il convient de rester prudent quant à l'interprétation des chiffres et des statistiques produits par le ministère de l'Intérieur et les administrations d'État pour justifier une politique migratoire à forte connotation sécuritaire. François Héran, titulaire de la chaire « Migrations et Sociétés » au Collège de France et directeur de l'Institut Convergences Migrations, met ainsi en garde contre l'instrumentalisation politique de telles données: "S'il y a 300000 irréguliers en France, qui est l'estimation la plus répandue mais sans doute inférieure à la réalité, les 23I suivis pour radicalisation, selon Gérald Darmanin, représentent

29. MEYER, Jean-Baptiste, "Le lien entre migration et terrorisme. Un tabou à déconstruire", Hommes \& migrations, $n^{\circ} 1315$, juillet-septembre 2016, pp. 49-57.

30. ADRAOUI, Mohamed-Ali, "La hijra au service d'un projet de rupture intégral dans le salafisme français", Ethnologie française, $n^{\circ}$ 4, 2017, pp. 649-658 ; ADRAOUI, Mohamed-Ali, "Partir au nom de Dieu? Islam et migration. L'exemple de la hijra dans le salafisme français", Migrations société, n 159-160, mai-août 2015, pp. 13-28. 
une proportion de I sur I300. Ce n'est pas négligeable mais ça invalide l’idée qu'il y aurait un lien nécessaire entre migrations irrégulières et radicalisation $»^{3 \mathrm{I}}$.

D'ailleurs, plusieurs études quantitatives en langue anglaise, recourant généralement à des modèles mathématiques, tendent à invalider cette relation entre immigration et terrorisme. D'abord celle de Vincenzo Bove et Tobias Böhmelt de l'Université de Warwick, qui s'est notamment basée sur la Global Terrorism Database croisant les informations disponibles sur les flux migratoires de 145 pays entre 1970 et 2000 : " Il ressort de leurs conclusions que si le terrorisme peut s'appuyer sur les réseaux migratoires pour recruter et répandre son idéologie, les flux de migrants en eux-mêmes ne contribuent pas à une montée du terrorisme dans les pays d'accueil. C'est même plutôt le contraire: plus l'immigration augmente, moins la menace terroriste est présente ${ }^{3^{2}}$. Une autre étude quantitative commanditée par le Cato Institute, think-tank libertarien américain partisan de la liberté de circulation, aboutit à des conclusions similaires: "Nous ne trouvons aucune preuve empirique suggérant que l'augmentation de la part d'immigrants de l'étranger est fortement corrélée à des taux plus élevés de terrorisme. Ces résultats valent pour les immigrants de pays à majorité musulmane et de pays déchirés par des conflits d'origine " ${ }^{33}$. Et les auteurs de conclure leur analyse : "L'implication en termes de politiques publiques est claire: des restrictions inspirées par la peur du terrorisme et l'immigration en provenance de pays en conflit sont malvenues ${ }^{34}$.

En France, les études statistiques sur le lien hypothétique entre immigration et terrorisme sont rares, pour ne pas dire inexistantes. En revanche, des enquêtes qualitatives conduites à partir de l'examen des dossiers des personnes fichées « $\mathrm{S}$ » (pour sûreté de l'État) ou d'entretiens semi-directifs menés avec des individus incarcérés dans les affaires récentes de jihadisme apportent un certain nombre de données pertinentes sur le caractère de plus en plus « endogène » des engagements terroristes ${ }^{35}$. L'une des plus éclairantes est sans doute

31. "Immigration et radicalisation: des chiffres rares dévoilés par Gérald Darmanin" [En ligne], L'Express avec l'AFP, 13 octobre 2020, https://www.lexpress.fr/actualite/societe/immigrationet-radicalisation-des-chiffres-rares-devoiles-par-gerald-darmanin_2136363.html.

32. AUDIGANE, Liv, "Une nouvelle étude démontre que l'immigration ne favorise pas le terrorisme" [En ligne], Slate.fr, 18 février 2016, http://www.slate.fr/story/114259/immigration-terrorisme. Pour les résultats complets de cette étude, voir BOVE, Vincenzo; BÖHMELT, Tobias, "Does Immigration Induce Terrorism?", The Journal of Politics, Volume 78, Number 2, April 2016, pp. 572-588.

33. FORRESTER, Andrew C. ; POWELL, Benjamin ; NOWRASTEHN Alex ; LANDGRAVE, Michelangelo, "Do Immigrants Import Terrorism?", CATO Working Paper, July 31, 2019, https://www.cato.org/publications/working-paper/do-immigrants-import-terrorism [Traduction de l'auteur].

34. FORRESTER, Andrew C. ; POWELL, Benjamin ; NOWRASTEHN Alex ; LANDGRAVE, Michelangelo, ibid. [Traduction de l'auteur].

35. SETTOUL, Elyamine, "Les jihadistes français : diversité des parcours, pluralité des réponses", Le Journal des psychologues, $\mathrm{n}^{\circ} 362$, novembre 2018, pp. 14-18 ; SETTOUL, Elyamine, "Le drapeau ou le djihad! Regard comparé sur les logiques d'engagement militaire et djihadiste", Confluences Méditerranée, $\mathrm{n}^{\circ}$ 106, Automne 2018, pp. 135-146; LIMAM, Wajdi, "Intervenants sociaux et radicalisation. Entre implicites publics et expérience sensible", VST - Vie sociale et traitements, $\mathrm{n}^{\circ} 138,2^{\mathrm{e}}$ trimestre 2018, pp. 86-92. 
celle dirigée par les chercheurs Xavier Crettiez et Romain Sèze qui, dans le cadre de la Mission Droit et Justice, ont eu directement accès aux personnes radicalisées en rapport avec la «cause jihadiste» et aussi avec la «cause basque », ce qui leur permet d'avoir un éclairage comparatif sur les parcours biographiques et le processus de socialisation à la violence des individus : "La menace terroriste est désormais interne; la notion de radicalisation cherche dès lors à mieux la saisir et comprendre comment des individus en apparence intégrés ont pu se retourner contre

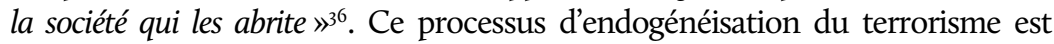
d'ailleurs confirmé par d'autres auteurs : «Si un temps le terrorisme islamiste était caractérisé par une menace exogène - où des forces extérieures viennent frapper lintérieur - aujourd'hui elle est beaucoup plus endogène : grâce à Internet notamment, des individus qui vivent (et qui parfois sont nés) sur notre sol, convertis ou religieux de naissance, se retrouvent en position d'être manipulés, première étape conduisant à la radicalisation. Pour cette raison, le lien de causalité direct et immédiat entre immigration et terrorisme est une vue de l'esprit, qui si hélas peut souffrir de tragiques contre-exemples, n'est plus, ou tout du moins ne doit plus être au cour de la réponse politique qu'il nous faut apporter ${ }^{37}$.

En définitive, si une réflexion approfondie sur la relation entre immigration et terrorisme doit être encouragée (aucun sujet n'est a priori tabou pour les sciences sociales $)^{38}$, il convient aussi de déconstruire la corrélation magique, très largement instrumentalisée par les hommes politiques et les experts sécuritaires qui aboutit à obscurcir le débat plus qu'elle ne contribue à le faire avancer. Pire, cette corrélation vient légitimer une sorte de synergie de la peur, une routinisation de la « gouvernementalité par l'inquiétude $»^{39}$, jouant sur les registres de l'émotion ${ }^{40}$, opérant par une série de glissements à la fois sémantiques et symboliques mais aux effets sociaux bien réels. À travers la mise en scène tragique de la corrélation magique entre immigration et terrorisme, les apprentis sorciers en viennent à enclencher un processus de «bouc-émissairisation » et de criminalisation de catégories entières de la

36. CRETTIEZ, Xavier ; SÈZE Romain (sous la direction de), Saisir les mécanismes de la radicalisation violente : pour une analyse processuelle et biographique des engagements violents, Rapport de recherche pour la Mission de recherche Droit et Justice, 2017, p. 9, http://www.giprecherche-justice.fr/wp-content/uploads/2017/08/Rapport-radicalisation_INHESJ_CESDIP_GIPJustice_2017.pdf.

37. ZAHAB, Jad, "Le lien de causalité direct entre immigration et terrorisme est une vue de l'esprit" [En ligne], Huffingtonpost, 28 septembre 2020, https://www.huffingtonpost.fr/entry/le-lien-decausalite-direct-entre-immigration-et-terrorisme-est-une-vue-de-lesprit_fr_5f71b761c5b61af20e787ccd.

38. MEYER, Jean-Baptiste, "Le lien entre migration et terrorisme. Un tabou à déconstruire", op. cit.

39. BIGO, Didier, "Sécurité et immigration : vers une gouvernementalité par l'inquiétude ?", Cultures \& conflits, art. cité.

40. ROBERT, Anne-Cécile, La stratégie de l'émotion, Montréal : Lux Éditeur, 2018, 148 p. 
société française ${ }^{41}$, qui va des sans-papiers et des demandeurs d'asile ${ }^{42}$ aux intellectuels dits «multiculturalistes » et «islamo-gauchistes »43, en passant par les immigrés légaux ${ }^{44}$, les musulmans ordinaires ${ }^{45}$, les nationaux français descendant des migrations post-coloniales et les habitants des banlieues populaires ${ }^{46}$, qui se retrouvent sur le banc des accusés, suspectés de complicité idéologique avec le terrorisme jihadiste: «pas coupables, mais responsables » selon la formule bien connue.

Marseille, le 2 décembre 2020

41. GIRARD, René, Le Bouc émissaire, (réédition), Paris : Le Livre de Poche, 2015, 320 p.

42. GAUTHIER, Catherine, "Asile et terrorisme. L'insidieuse érosion des statuts de réfugié et de bénéficiaire de la protection subsidiaire", Revue des droits et libertés fondamentaux, $n^{\circ} 48,2019$. http://www.revuedlf.com/droit-ue/asile-et-terrorisme-linsidieuse-erosion-des-statuts-derefugie-et-de-beneficiaire-de-la-protection-subsidiaire.

43. COLLECTIF, "Les libertés sont précisément foulées aux pieds lorsqu'on en appelle à la dénonciation d'études et de pensée", Le Monde, 5 novembre 2020. Cette tribune a été rédigée en réaction au texte inquisiteur signé par 100 personnalités du monde académique, la majorité d'entre elles à la retraite: "Une centaine d'universitaires alertent : 'Sur l'islamisme, ce qui nous menace, c'est la persistance du déni'", Le Monde, 31 octobre 2020.

44. VIGNAL, François, "'L'immigration d'aujourd'hui, ce sont les terroristes de demain': tollé au Sénat après les propos du sénateur Masson" [En ligne], Public Sénat, 14 octobre 2015, https://www.publicsenat.fr/lcp/politique/l-immigration-d-aujourd-hui-terroristes-demaintolle-senat-propos-senateur-masson-1082.

45. NEUVILLE, Héloïse de ; LE NORMAND, Xavier, "'Séparatisme', le malaise des Français musulmans" [En ligne], La Croix, 13 octobre 2020, https://www.la-croix.com/Religion/Separatisme-malaiseFrancais-musulmans-2020-10-13-1201119231; voir aussi le communiqué de la CONFÉDÉRATION GÉNÉRALE DU TRAVAIL, "Projet de loi contre le séparatisme : une nouvelle loi contre les musulmans", 08 octobre 2020, https://www.cgt.fr/actualites/projet-de-loi-separatisme-unenouvelle-attaque-contre-les-musulmans.

46. JARDIN, Antoine, "Banlieues, le berceau du terrorisme?", Sciences humaines, $n^{\circ} 279$, mars 2016, p. 27. 
